\title{
Research on Characteristics of Airline Network Based on Passenger Flow
}

\author{
Xu Shunzhi ${ }^{*}, 1,2$, Gao Qiang ${ }^{1}$ and Zhu Jinfu ${ }^{1}$ \\ ${ }^{1}$ College of Civil Aviation, Nanjing University of Aeronautics and Astronautics, Nanjing, 210016, P.R. China; ${ }^{2}$ Jincheng \\ College, Nanjing University of Aeronautics and Astronautics, Nanjing, 211156, P.R. China
}

\begin{abstract}
To reflect the scale-free characteristics of airline network, generating $\mathrm{n}$ city nodes randomly while the degree distribution exponent is located between 1 and 3, by constructing network edges between nodes, calculating the passenger flow of each network edge, building the relationship of fares to node distance and aircraft type to node type, we propose the mathematical model of airline network revenue, total cost and total profit. Finally, we simulate the airline network profit model and analyze the experimental results, which show that: under the influence of the total number of nodes, the number of hub nodes, the capacity of the hub node and fares, the airline network profit changes in a certain regularity with the change of passenger flow.
\end{abstract}

Keywords: Airline flow, Airline network, Airline profit, Economic characteristics, The degree distribution exponent.

\section{INTRODUCTION}

The airline network is composed of the essential factors which include airport, airline aircraft, etc., wherein the airport and airline decide the distribution of air transport space and the ground and air support capacity of air transportation, aircraft completes the spatial displacement of passengers and freight through the airline from one airport to another; at the same time, the number of airports and aircrafts is the time evolution, airline will also present dynamics because of the capacity constraints, the budget and the market demand (OD). Airline network belongs to the typical complex system, which has the scale-free connection characteristics [1].

Scale-free network [2] reveals the universality of growth and preferential attachment mechanism in the complex network of self-organization evolution and the importance of power-law. With the appearance of the scale-free network model, there appeared the MoriTanaka method [3], the rateequation method [4], the master-equation method [5], the numerical calculation method of Markov chain [6,7], all of which can be used to calculate the distribution of stability degree. The research on the hub node is mainly concentrated in the virus spread in the network, the network robustness and influence of the vulnerability [8-12]. These studies researched network characteristics from the angle of mathematics, and it would not reveal the real world networks phenomenon, therefore there appeared the study of weighted network and space network [7, 13-14], which have been applied in the complexity $[7,15]$ and optimization theory [1619] of airline network. The above researches are lack of insufficient consideration of political and economic factors of airline network; they do not well reflect running characteristics of airline network and far away to application.

*Address correspondence to this author at the College of Civil Aviation, Nanjing University of Aeronautics and Astronautics, Nanjing, 210016, P.R. China; Tel: 15335198002; E-mail: xsz166126@126.com
This paper allows the node among network which is not connected and repeated and a node connecting the others randomly according to the node degree value; as a result, there is no isolated node and no redundant degree value of each node. By constructing the relationship between fares and airline distance, airline passenger flow and airline distance, airplane type and node type, we researched airline network profit changes with the change of airline passenger flow under the influence of the total number of nodes $n$, the capacity of the hub node, reflecting the economic characteristics of airline network and it is important for the airline to improve the efficiency of transportation, to reduce the transportation cost and to optimize the network design.

\section{ESTABLISHMENT OF AIRLINE NETWORK AND ITS PROFIT MODEL}

$\mathrm{N}$ (city) node $\operatorname{node}\left(s_{i}, x_{s_{i}}, y_{s_{i}}, K_{s_{i}}, t_{s_{i}}\right)$ and $n \times n$ matrix of OD flow $O D_{-} \operatorname{flow}\left(s_{i}, s_{j}\right)$ were randomly generated. Wherein, $s_{i}$ expresses as the $\mathrm{i}$ node $(\mathrm{i}=1,2,3 \ldots \mathrm{n})$, The $x_{s_{i}}$ and $y_{s_{i}}$ identify the ordinate and abscissa of the i node, $K_{s_{i}}$ expresses the value of the $\mathrm{i}$ node degree, $t_{s_{i}}$ expresses the kind of the i node $\left(t_{s_{i}}=0,1\right.$. if $s_{i}$ is the hub node, $\left.t_{s_{i}}=1\right)$; the path between node $s_{i}$ and node $s_{j}$ can be the direct path transfer $0\left(s_{i}, s_{j}, d\left(s_{i}, s_{j}\right)\right)$ (Wherein, $d\left(s_{i}, s_{j}\right)$ expresses the distance of network edge $\left.s_{i} s_{j}\right)$, a once transfer path transfer $1\left(s_{i}, s_{m}, s_{j}, d\left(s_{i}, s_{j}\right)\right)$ and the secondary transfer path transfer $2\left(s_{i}, s_{m}, s_{n}, s_{j}, d\left(s_{i}, s_{j}\right)\right)$; the direct_ration as the proportion of the direct path flow $d_{-} f \operatorname{low}\left(s_{i}, s_{j}\right)$ accounted for the node flow $O D_{-} \operatorname{flow}\left(s_{i}, s_{j}\right)$, that is, 
$d_{-} \operatorname{flow}\left(s_{i}, s_{j}\right)=O D_{-} f \operatorname{flow}\left(s_{i}, s_{j}\right) *$ direct_ration; the redistribution edge flow $\operatorname{linkflow}\left(s_{i}, s_{l}\right)$ can be described as: $\operatorname{linkflow}\left(s_{i}, s_{l}\right)=\operatorname{linkflow}\left(s_{i}, s_{l}\right)+t_{-} \operatorname{flow}\left(s_{i}, s_{j}\right)[\operatorname{total} 1 \div$ total $]$ (wherein, $\quad t_{-} \operatorname{flow}\left(s_{i}, s_{j}\right)=O D_{-} \operatorname{flow}\left(s_{i}, s_{j}\right) *(1-$ direct _ration $), \quad$ total $1=\left[1 / d\left(s_{i}, s_{l}\right)+1 / d\left(s_{l}, s_{j}\right)\right], \quad$ total $=[1 / d$ $\left.\left.\left(s_{i}, s_{l}\right)+1 / d\left(s_{l}, s_{j}\right)+1 / d\left(s_{i}, s_{m}\right)+1 / d\left(s_{m}, s_{n}\right)+1 / d\left(s_{n}, s_{j}\right)\right]\right)$; the fares particularly direct fares $O D_{-} p\left(s_{1}, s_{2}\right)=d\left(s_{i}, s_{j}\right) *$ relation_ration (wherein, $\left.d\left(s_{i}, s_{j}\right)=\sqrt{\left(x_{s_{1}}-x_{s_{2}}\right)^{2}+\left(y_{s_{1}}-y_{s_{2}}\right)^{2}}\right)$; the corresponding transit airline fares transfer $\ldots\left(s_{i}, s_{j}\right)=$ $O D_{-} p\left(s_{i}, s_{j}\right) *$ transfer_ration; based on the airlines are composed of different types of nodes, the corresponding types of airplane and cost per km can be shown as: $u_{1}, u_{2}$, $a p_{-} t\left(u_{1}\right), a p_{-} t\left(u_{2}\right), a p_{-} c\left(u_{1}\right), a p_{-} c\left(u_{2}\right)$.

Therefore, the total revenue $\left(t_{-}\right.$revenue $\left.(n)\right)$, the total variable cost $\left(c_{-} \cos t(n)\right)$, the total fixed cost $\left(s_{-} \cos t(n)\right)$, the total cost $\left(t_{-} \cos t(n)\right)$ and profit (net_profit $\left.(n)\right)$ of airline network can be expressed as:

$$
\begin{aligned}
& t_{-} \text {revenue }(n)=\sum_{i=1}^{n} \sum_{j=1}^{n}\left[d_{-} f\left(s_{i}, s_{j}\right) * O D_{-} p\left(s_{i}, s_{j}\right)+\right. \\
& \left.t_{-} f\left(s_{i}, s_{j}\right) * \text { transfer_ } p\left(s_{i}, s_{j}\right)\right] \\
& c_{-} \cos t(n)=\sum_{i=1}^{n} \sum_{j=1}^{n} \operatorname{linkflow}\left(s_{i}, s_{j}\right) * d\left(s_{i}, s_{j}\right) \\
& s_{-} \cos t(n)=\sum_{l=1}^{2} \sum_{i=1}^{n} \sum_{j=1}^{n} \frac{\operatorname{linkflow}\left(s_{i}, s_{j}\right)}{a p_{-} t\left(u_{l}\right)} * a p_{-} c\left(u_{l}\right) * d\left(s_{i}, s_{j}\right) \\
& t_{-} \cos t(n)=c_{-} \cos t(n)+s_{-} \cos t(n) \\
& n e t_{-} \operatorname{profit}(n)=t_{-} \operatorname{revenue}(n)-t_{-} \cos t(n)
\end{aligned}
$$

With the given change rate of airline passenger flow, we researched the relationship between airline network profit and the airline passenger flow while the parameter scenarios are determined, and the technical route is shown in Fig. (1).

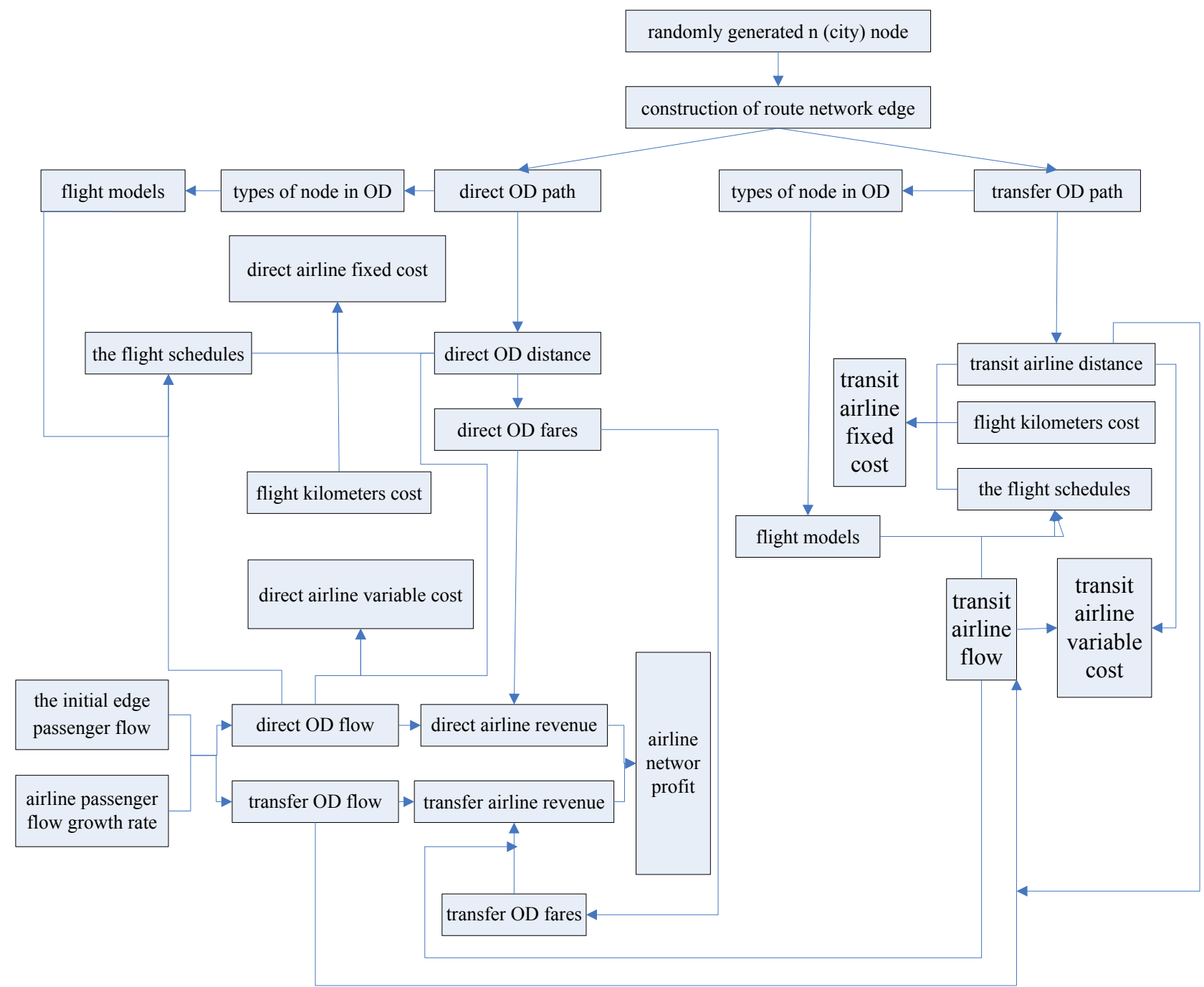

Fig. (1): The technical route. 


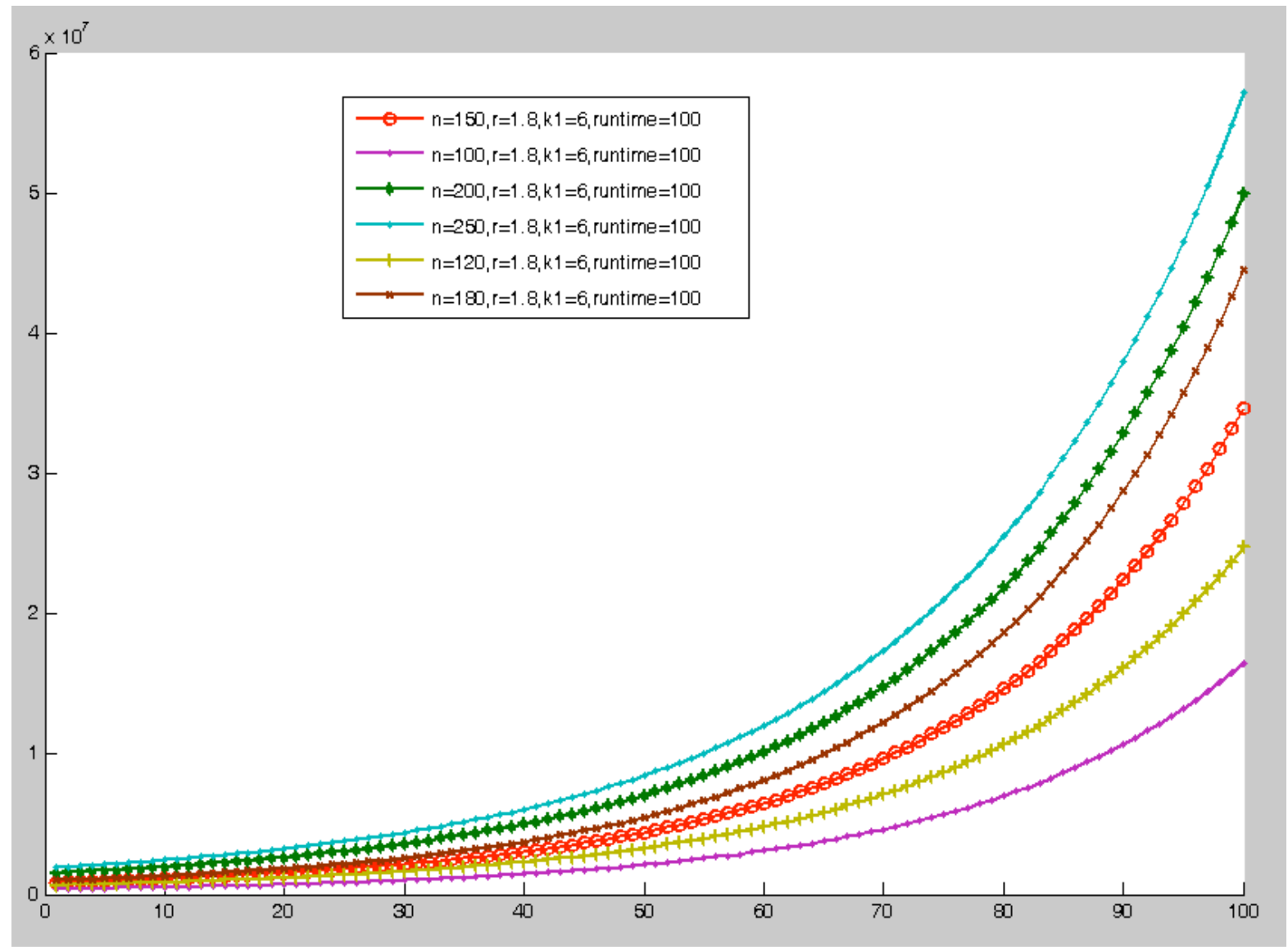

Fig. (2): The relationship between airline network profit and the increasing times of passenger flow based on the given number of network node.

\section{SIMULATION STUDY}

In order to meet the simulation research and to reveal the economic characteristics of airline network largely, we assume the value and the range of some parameters after the analysis of the information of related civil aviation enterprises and institutions. We assume the degree value of hub node from $0.2 m_{-} d$ to $0.53 m_{-} d$ ( $m_{-} d=n-1$, the maximum degree value of the node) based on the thought of the dominance of hub node and the node capacity restriction in the airline network; the randomly generated OD flow are no more than 2500 people, that is $O D_{-} \operatorname{flow}\left(s_{i}, s_{j}\right)$ $\in[0,2500]$; In addition, we also give the value of certain variables directly, direct_ration $=0.6$; relation_ration $=$ $1.5 ;$ transfer_ration $=0.6 ; d t_{-} m=0.05 ; t_{-} m=0.02$; $d_{-} m=0.04 ; a p_{-} t\left(u_{1}\right)=350 ; a p_{-} c\left(u_{1}\right)=0.001 ; a p_{-}$ $t\left(u_{2}\right)=200 ; a p_{-} c\left(u_{2}\right)=0.0007$.

\subsection{The Study of the Relationship Among Airline Net- work Profit, the Total Numbers of Node, Airline Passen- ger Flow and Fares}

Airlines always look forward to remain invincible in the transportation market, besides the existing airlines, they so actively explore new markets to excavate potential customers and to win more profit. We select the degree distribution exponent $\mathrm{r}$ equal to $1.8(r=1.8)$, the hub node number is equal to $6(k=6)$, the network node number $(n)$ is respectively equal to $100,120,150,180,200$ and 250 , the maximum times of passenger flow increase is equal to 100 ( $\max$ runtime $=100$ ), then we researched the airline network profit with the times of passenger flow increase of different kinds of airlines in the given network node number, as shown in Fig. (2).

Fig. (2). shows that when the other variables remain unchanged, there are more network nodes, and more airline network profit. The change of the airline network profit occurs with the redistribution of $O D_{-} f l o w\left(s_{i}, s_{j}\right)$ and the re-

value of direct_ration, which is from 0.6 to 0.7 , as shown in Fig. (3).

Fig. (3). shows that the above conclusion of the more network nodes the more airline network profit has not appeared when direct_ration is equal to 0.7 (direct ration $=0.7$ ), and the airline network profit according to 150 nodes $(n=150)$ is less than the profit according to 120 network nodes $(n=120)$. This is because the airline will get high revenue due to more passenger flow of the direct airlines and the more direct flights cost leads to the less passenger flow of the transit airlines. In fact, fares and the proportion of direct airline passenger flow and transit airline passenger flow are inseparable, the above two should be 


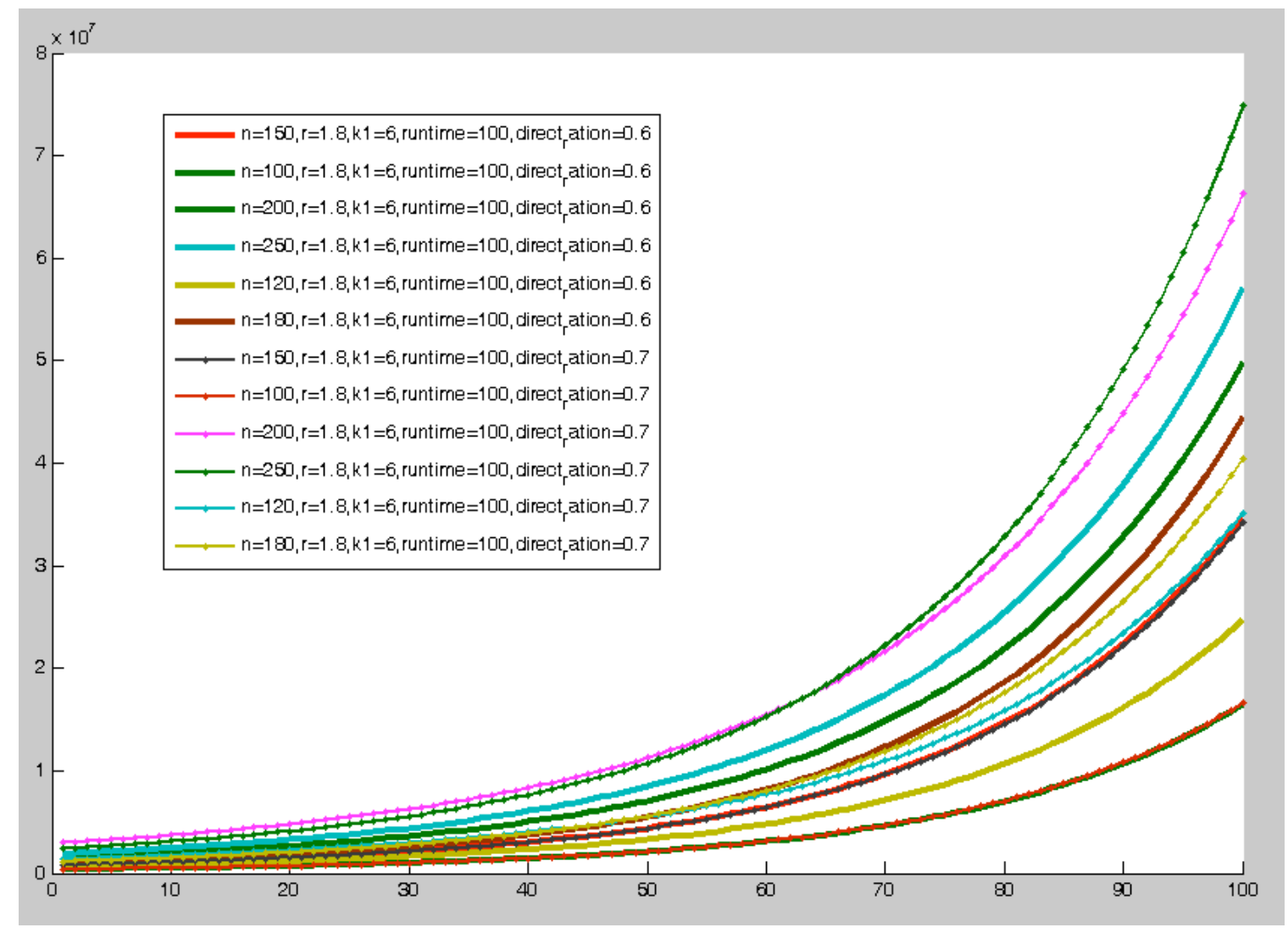

Fig. (3). The relationship between airline network profit and the increasing times of passenger flow based on the OD flow of network edge.

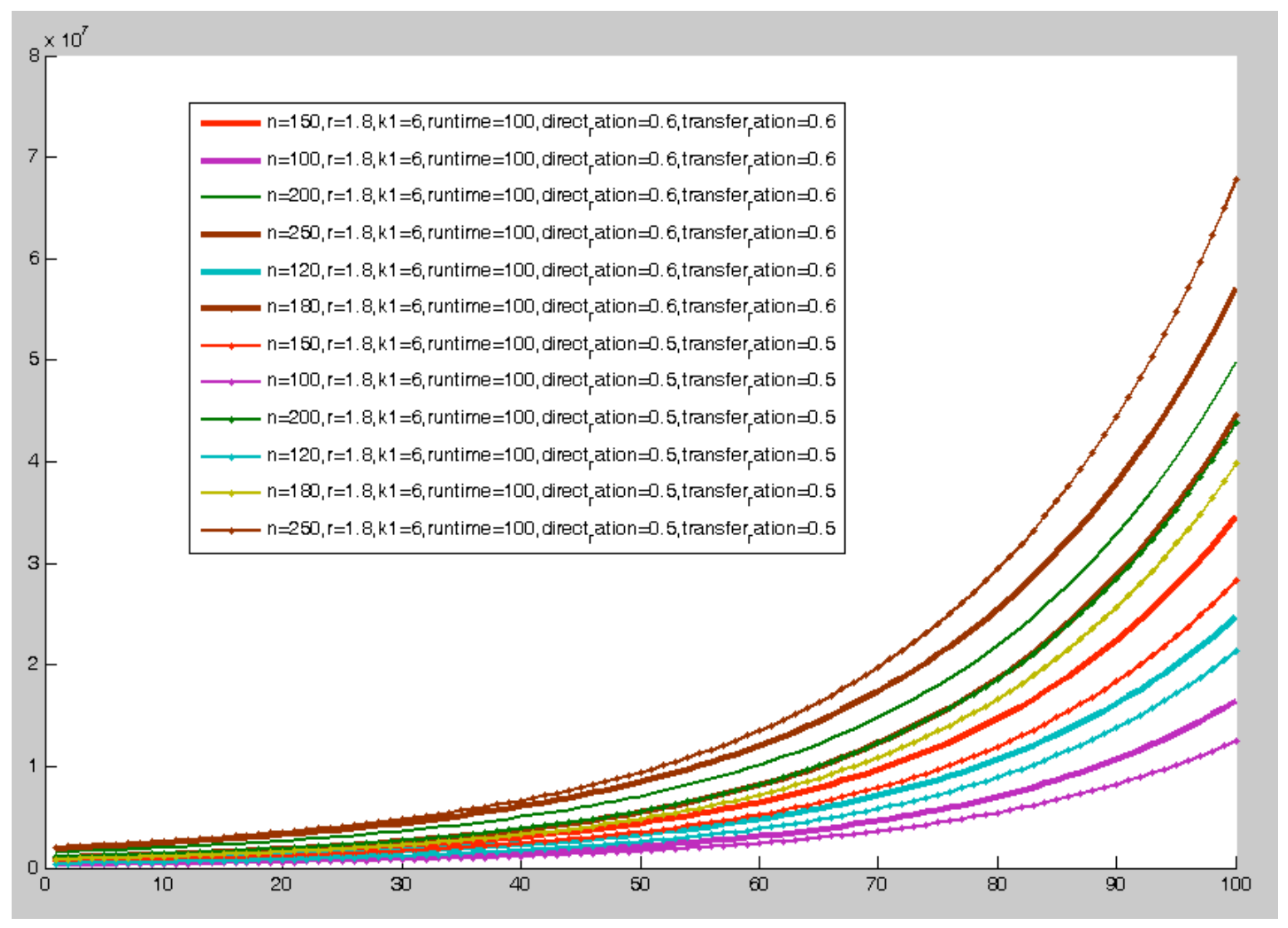

Fig. (4). The relationship between airline network profit and the increasing times of passenger flow based on the OD flow of network edge and fares. 


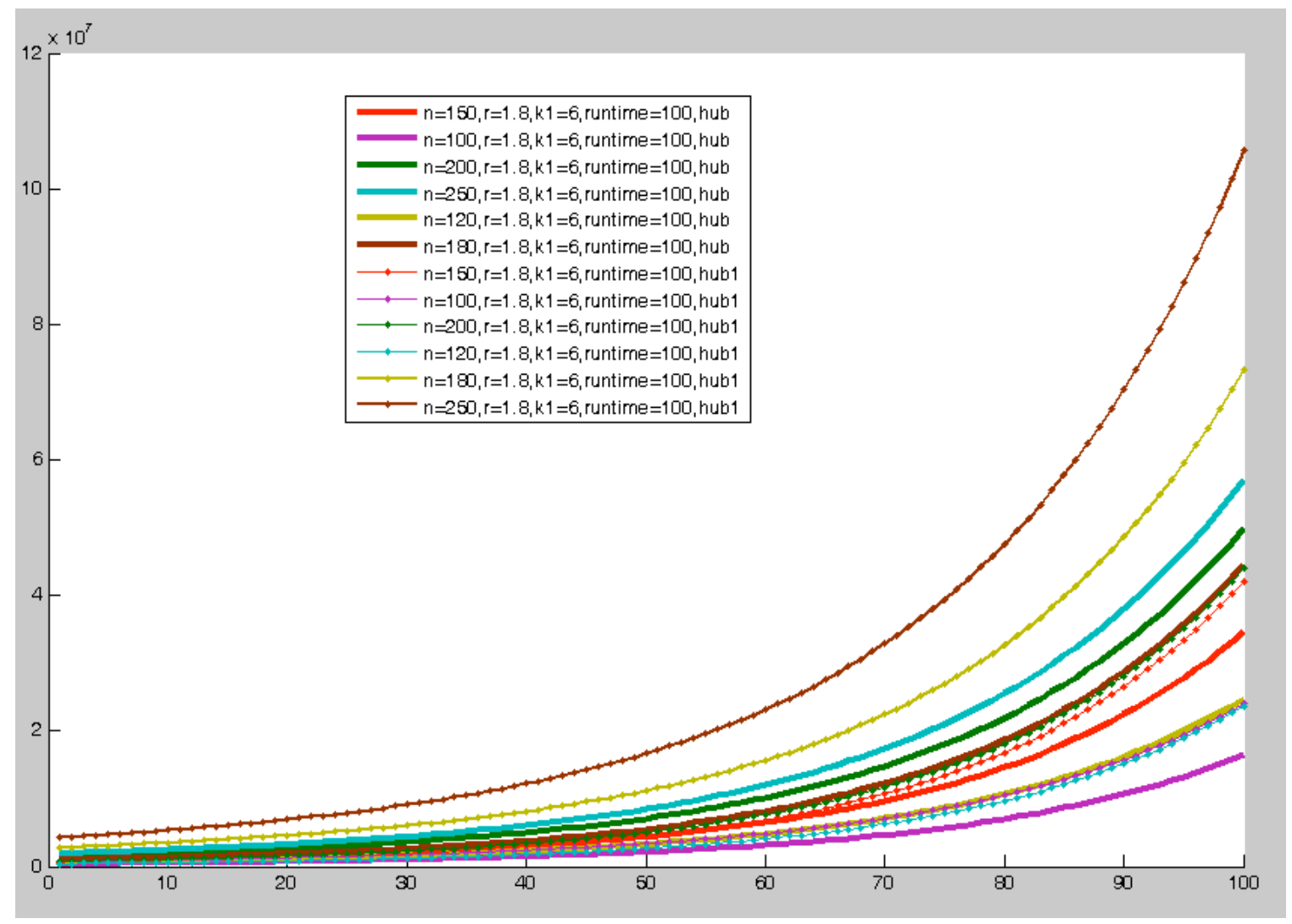

Fig. (5): The relationship between airline network profit and the increasing times of passenger flow based on the capacity limit of hub node.

integrated to research airline network profit, the change of the airline network profit occurring with the direct_ration value from 0.6 to 0.5 and the transfer_ration value from 0.6 to 0.5 , as shown in Fig. (4).

Fig. (4). shows that with the decrease of direct airline passenger flow and increase in transit airline fares, airlines will save flights cost for the more transit airline passenger flow and will get low revenue for the less direct airline passenger flow except for the condition of $\mathrm{n}$ being equal to $180(n=180)$. Moreover, the airline network profit decreases because the reduction of airline network revenue is greater than the saved cost of airline network. On the contrary, the airline network profit increases because the reduction of airline network revenue is less than the saved cost of airline network when $\mathrm{n}$ is equal to $180(n=180)$.

Through the above analysis, if we want to analyze how the number of total network nodes, the value of direct_ration and transfer_ration influence the airline network profit when the other variables remain unchanged, we should consider these three factors together.

\subsection{The Study of the Relationship between Airline Net- work Profit and the Capacity of the Hub Node}

To describe the capacity of hub node how to impact on the airline network profit, we select the degree distribution exponent $\mathrm{r}$ which is equal to $1.8(r=1.8)$, the hub node number is equal to $6(k=6)$, the degree value of hub node is respectively from $0.2 m_{-} d$ to $0.53 m_{-} d$ (hub1), from 0 to $m_{-} d$ (hub2), the maximum times of passenger flow increase is equal to 100 ( $\max$ runtime $=100)$, then we researched the airline network profit with the times of passenger flow increase of different kinds of airlines in the condition of the given range of the hub node degree value, as shown in Fig. (5).

It can be observed from Fig. (5)., when the other variables remain unchanged, the hub node can connect with more nodes if there is no capacity limit on the hub node, as a result, the range of the degree value of network nodes is bigger, the corresponding airline network profit is larger than the profit with the capacity limit on the hub node.

Contrary to the above conclusion of the more nodes, there is more airline network profit when the other variables remain unchanged. If there is no capacity limit on the hub node, airline network profit according to 250 nodes $(n=250)$ will be less than the profit according to 180 network nodes $(n=180)$. From the simulation result, the degree value of hub node is very big, which is equal to 176,174 , $173,86,24$ and 11 , respectively when $\mathrm{n}$ is equal to 180 $(n=180)$, this airline network shows more characteristics of the hub-and-spoke airline network, which reflects the motivation about reason why airlines strive to build the hub-andspoke airline network.

\section{CONCLUSION}

This paper focuses on the study of variation of airline network profit with the airline passenger flow changes based on the constraint of factors, such as the number of network 
node, the number of hub node, the passenger flow of network edge, fares and the capacity of network hub node. Although it is based on certain assumptions, we still can obtain some valuable conclusions, for example, the airline network shows more characteristics of the hub-and-spoke airline network while there is no capacity limit on the hub node, but the research does not distinguish which nodes belong to the hub node, which airlines belong to the key routes, besides, it also does not concern about the cost of opening new airlines and the virtual network structure, which is used to meet larger passenger flow, which will be the focus of future study.

\section{CONFLICT OF INTEREST}

The authors confirm that this article content has no conflict of interest.

\section{ACKNOWLEDGEMENTS}

This research was funded by the National Natural Science Foundation of China (No. 71171111), the Colleges and Universities Natural Science Research Project in Jiangsu Province (No. 14KJD580004), the Ordinary University Innovative Research Projects in Jiangsu Province (CXLX11 0208). This research was also sponsored by Qing Lan Project.

\section{REFERENCES}

[1] R. Guimera, S. Mossa, A. Turtschi, and L.A.N. Amaral, "The worldwide air transportation network: Anomalous centrality, community structure, and cities' global roles", Proceedings of the $\mathrm{Na}$ tional Academy of Sciences, vol. 102, pp. 7794-7799, 2005.

[2] A. L. Barabási, and R. Albert, "Emergence of scaling in random networks", Science, vol. 286, pp. 509-512, 1999.

[3] P. L. Krapivsky, S. Refiner, and F. Leyvraz, "Connectivity of growing random networks”, Physical Review Letters, vol. 21, pp. 4629-4632, 2000.

[4] S. N. Dorogovtsev, J. F. Mendes, and A. N. Samukhin, "Structure of growing networks with preferential linking", Physical Review Letters, vol. 21, pp. 4633-4636, 2000.
[5] D. H. Shi, Q. H. Chen, and L. M. Liu, "Markov chain-based numerical method for degree distributions of growing networks", Physical Review E, vol. 71, pp. 036140, 2005.

[6] Q. H. Chen, and D. H. Shi, "Markov chains theory for scale-free networks", Physica A: Statistical Mechanics and its Applications, vol. 360, pp. 121-133, 2006.

[7] A. Barrat, M. Barthélemy, and A. Vespignani, "Weighted evolving networks: coupling topology and weight dynamics", Physical Review Letters, vol. 22, pp. 228701, 2004.

[8] R. Pastor-Satorras, and A. Vespignani, Epidemics and Immunization in Scale-Free Networks, Handbook of graphs and networks. from the genome to the internet, vol. 1, pp. 111-130, 2005.

[9] R. Albert, H. Jeong, and A. L. Barabási, "Error and attack tolerance of complex networks", Nature, vol. 6794, pp. 378-382, 2000.

[10] M. Wolfson, A. Budovsky, and R. Tacutu, "The signaling hubs at the crossroad of longevity and age-related disease networks", The International Journal of Biochemistry and Cell Biology, vol. 3, pp. 516-520, 2009.

[11] R. Yang, B.H. Wang, and J. Ren, "Epidemic spreading on heterogeneous networks with identical infectivity", Physics Letters A, vol. 3, pp. 189-193, 2006.

[12] X. F. Wang, and G. Chen, "Pinning control of scale-free dynamical networks", Physica A: Statistical Mechanics and its Applications, vol. 3, pp. 521-531, 2002.

[13] S. P. Borgatti, "Centrality and network flow", Social networks, vol. 1, pp. 55-71, 2005.

[14] S. Boccaletti, V. Latora, and Y. Moreno, "Complex networks: Structure and dynamics", Physics reports, vol. 4, pp. 175-308, 2006.

[15] A. Reggiani, P. Nijkamp, and A. Cento, Connectivity and Competition in Airline Networks: A Study of Lufthansa's network, The Network Experience, Springer: Berlin, vol. 3, pp. 111-122, 2011.

[16] X.H. Wu, J.F. Zhu, and W.W. Wu, "A weighted network evolving model with capacity constraints", Science China Physics, Mechanics and Astronomy, vol. 9, pp. 1619-1626, 2013 (in Chinese).

[17] W. Ge, J.F. Zhu, and W.W. Wu, "Design of spider webairline network model", Journal of Transportation Systems Engineering and Information Technology, vol. 4, pp. 172-177, 2012.

[18] X.H. Wu, J.F. Zhu, and W.W. Wu, "Relative interval robust optimization of airline network designing", Journal of Systems Engineering, vol. 1, pp. 69-77, 2012.

[19] X.H. Wu, J.F. Zhu, and W.W. Wu, "Interval robust optimization of airline network designing", Journal of Southwest Jiaotong University, vol. 3, pp. 559-564, 2013. 